\title{
INFORMATION GEOMETRY OF DIFFERENTIALLY FED ARTIFICIAL NEURAL NETWORKS
}

Manjunath.R, Dr.K.S.Gurumurthy

(Dept.of EC \& CSE UVCE,Bangalore)

e-mail:manju_r_99@yahoo.com

\begin{abstract}
Anew class of neural networks with differential feed back are presented.The different orders of differential feedback form a manifold of hyperplanes, one among them called eigen plane corresponding to $\infty$ order feedback.In this paper information geometry is used to explore the interesting properties of this plane.
\end{abstract}

Keywords:-Differential feedback, ANN Information geometry

\section{INTRODUCTION}

Information geometry uses a family of probability distributions to compute quantities related to pdf including mutual interactions. It was used to study multilayer perceptrons [6].

A family of distributions called exponential family has the pdf

$P(y, \theta)=\exp \left\{\Sigma \theta_{i} k_{i}(y)-\psi(\theta)\right\}-\cdots-\cdots(1)$

Where $\theta$ is the cosystem, $k=k_{i}(y)$ are adequate functions of $y, \psi$ the offset. The exponential family forms $\alpha= \pm 1$ flat manifold. For this Riemannchristoffel curvature vanishes identically. This is non Euclidean space. For this manifold, there exists $\alpha= \pm 1$ affine cosystem. This is because log of pdf is linear in $\theta$. For any two distributions $p(y)$ and $q(y)$ the geodesic connecting them is given by

$\log (p(y, t))=(1-t) \log p(y)+t \log q(y)-\psi(t)---(2)$

A family of Divergence measures namely $\alpha$ divergence , is associated with the manifold of pdf[7]. The $\alpha=-1$ divergence is known as Kullback divergence. These divergence functions give a Reimannian metric to the manifold of pdf.

The networks are represented by a set of parameters called weights $\theta=w_{i j}$.A family of networks parameterized by $\theta$ forms a manifold, $\theta$ playing the role of a cosystem. This is shown in Fig. 1 The position of a network in this co system is shown in the figure. In this paper, it is shown that they form hyperplanes for different choices of feedback. The amount of feedback is proportional to Kullback divergence distance i.e., planes are Kullback distance apart. The kullback divergence of pdf $p$ from pdf $q$ is given by

$\mathrm{p}^{*} \log (\mathrm{p} / \mathrm{q}) \cdots(3)$

In this paper, different orders of differential feedback form a manifold of hyperplanes and are related to manifolds of pdfs. The distance between them and the trajectory of a point on these planes is explored.

In secII, concept of differentially fed neural networks is explored. In sec III information geometry of differential feedback is given. Section IV concludes with the properties of differential feedback

\section{FORMALISM OF DIFFERENTIALLY FED ANN}

The output $y$ of a neural network but for the nonlinearities can be written as

$$
y=\text { Lwixi.----(4) }
$$

where $\mathrm{xi}$ are the inputs wi, the corresponding weights. The space spanned by weight vector for different inputs is a hyperplane as shown in the Fig. 1.

The important factor is weight cannot span the entire input space, whatever may be the training mode. Again the linearity of the output (1) may be viewed as a particular case of ARMA

$Y(n+1)=b 0 y(n)+b 1 y(n-1)+\ldots . .+a 0 x n+\ldots----(5)$

Where b0.. and a0.. are constants. The auto regressive terms $b 0 . . . b n$ may be realized using an implied differential feedback [1]. With differential feedback it has been found out[1] that the no of iterations required for training is reduced as shown in the table I.XOR gate is considered for simulation. Gaussian distributed random input with seed value 1000 is taken as input. With I order different feedback, the output may be written as :

Ewixi +b1y1-----(6) 
$y 1$ being the $I$ order differential. This equation once again represents a plane parallel to $\Sigma$ wixi. Thus the set of differentially fed ANNs form a manifold of parallel planes, with $\infty$ order feedback being the plane with zero error.
In II order differential feedback system, the two differential terms can be replaced by a single term. Extending this principle, the $\infty$ terms of $\infty$ order differential feedback can be replaced

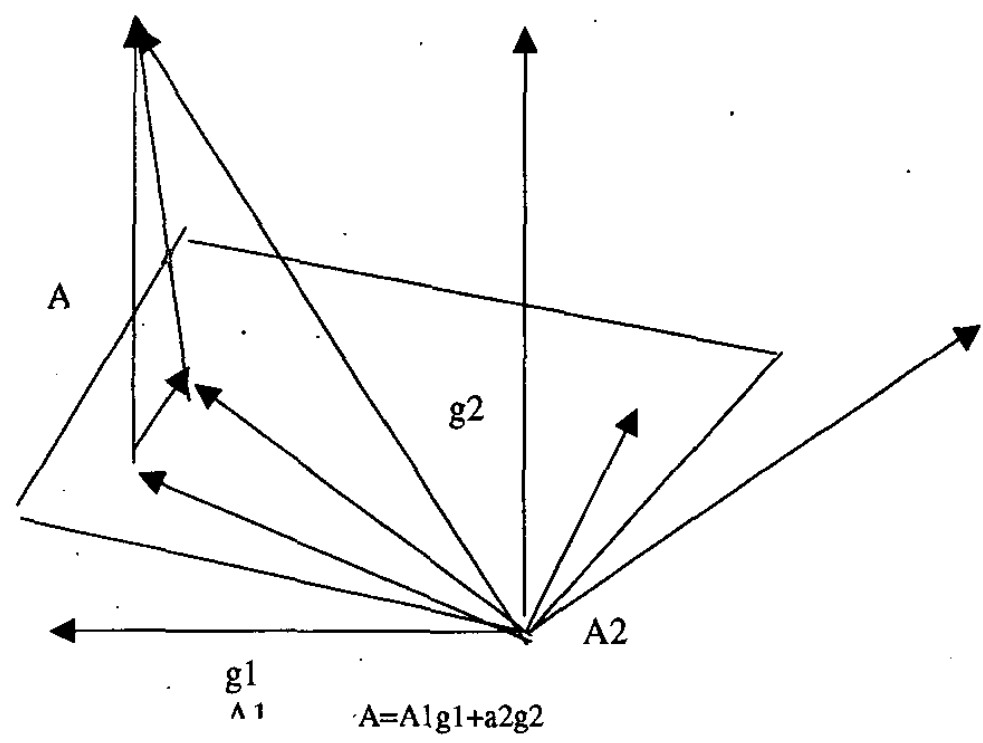

Fig.1.A=projection of $\mathrm{F}$ on to the subspace spanned by weight vector

Table I.Performance with feedback

\begin{tabular}{|l|l|l|}
\hline Order of differential & Square error & Iterations \\
\hline No feedback & 18 & 1156 \\
\hline I order & 18 & 578 \\
\hline II order & 18 & 289 \\
\hline
\end{tabular}

Table 2..Performance with II order feedback

\begin{tabular}{|l|l|l|}
\hline Order of differential & Square error & Iterations \\
\hline II irder Feedback & 18 & 578 \\
\hline Equivalent Output feedback & 18 & 578 \\
\hline
\end{tabular}

Also, simulation results of table II show that two terms of II order differential feedback i.e., y2-y1 and $\mathrm{y} 1-\mathrm{y} 0$ can be replaced by a single equivalent plane represented by

$\mathrm{Weq}=(\mathrm{w} 1 * \mathrm{extra}+\mathrm{w} 2 * \mathrm{iextra} 1) / \mathrm{y} 0--(7)$ by a single term.

This is termed as eigen plane which is the practical way of generating lowest error. Now the differential feedback becomes 
$\mathrm{dy} / \mathrm{dt}+\mathrm{d}^{2} \mathrm{y} / \mathrm{dt}^{2}+\ldots----(8)$

Taking $\mathrm{Z}$ transform, \& then the inverse,

yeq=IZT $\{Y(z) /(1-z)\}-----(9)$

\section{INFORMATION GEOMETRY OF DIFFERENTIAL FEED BACK}

For less error, the plane spanned by the weight vectors should be as close as possible to the eigen plane.

When I order differential feedback is given, the new plane is given by

Ynew $=$ Swixi+a*yold----..---(10)

Which is a parallel plane. To start with, set Yold $=0 . S o, y=\sum$ wixi. Since error varies asymptotically with order, the gap between parallel planes decreases and the infinite order plane coincides with the eigen plane. If still more feedback is given, error increases further as shown in fig. 3

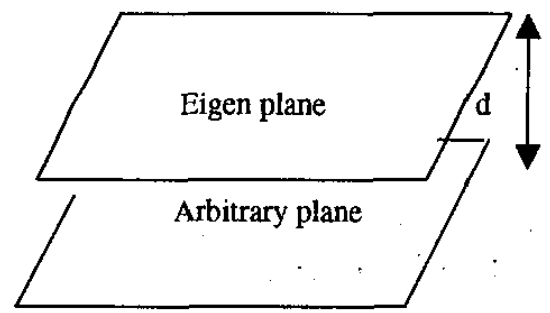

To show that the entropy is minimum on the eigen plane, consider the exponential family as

given in [3]. The error may be assumed to be Gaussian distributed. In Gaussian distribution with Zero mean, The pdf can be written as

$p(x)=\exp \left((\right.$ energy of $\left.x) / \sigma^{2}\right),-----(11)$

The error energy of a plane $x$ may be written as ( $x$ $d)^{2}$. ( $\left.x-d\right)$ being the distance of $x$ from eigen plane d (or the actual value). The entropy of such a distribution takes minimum value when $(x-d)=0$ i.e. entropy is min when the plane reaches the eigen plane as shown in fig. 4 .

The Natural learning algorithm is given by[2]

$\theta(t+1)=\theta(t)-\eta G^{-1} \quad \cdots-(12)$

i.e.,newplane $=$ oldplane+deviation This shows that the repeated learning in gradient descent algorithm shifts the planes towards the eigen plane in the same way the diff feed back will do ,but fails to reach it because eigen plane does not belong to the space spanned by inputs alone.

Fig.2.Differential feedback planes

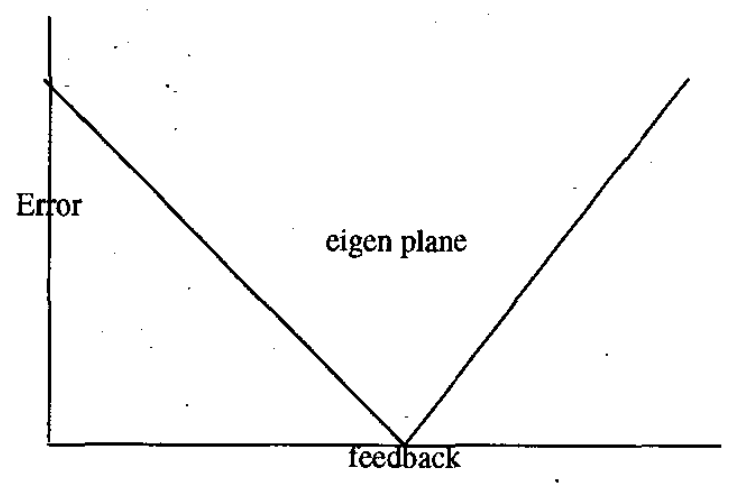

Fig.3.Feedback.Vs.Error 


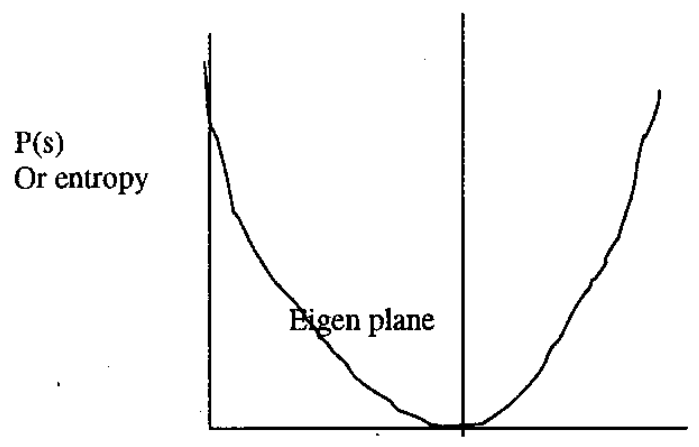

Order of diff FB

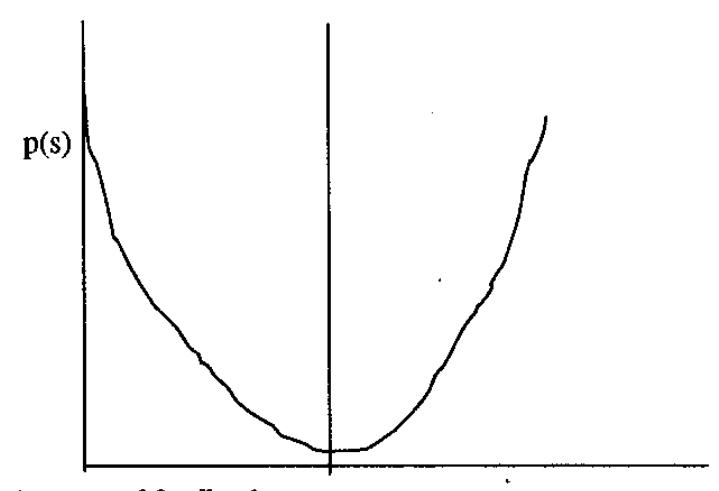

Amount of feedback

Fig.4 Entropy Vs.planes and feedback

Actual space

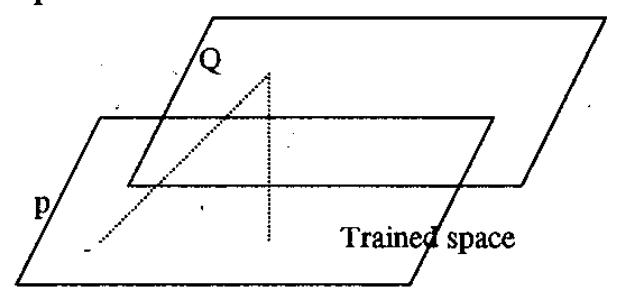

Fig.5.Affine transportation

The deviation of any plane from the eigen plane is given by Kullback Divergence.The Kullback divergence of $q$ from $p$ is given by

$D(p \| q)=\int p(s) \log (p(s)) / q(s) d \lambda(s) \cdots(13)$

Here $q(s)$ is the prob. Distribution of eigen plane $p(s)$ is prob distribution of some plane so that $D$ is the deviation wrt eigen plane. At eigen plane $p=q$ so $\mathrm{D}$ (pliq) $=0$

The important thing here is the distance between hyper planes (wrt eigen plane) is reflected as joint or conditional pdf

Consider 2 planes $\mathrm{p} 1(\mathrm{x})$ and $\mathrm{p} 2(\mathrm{x})$ as two nearby planes.one being the eigen plane.

The amount of feedback to be given =weigen*yeq( $\mathrm{t}$ )

But this distance is the Kullback divergence.

\section{Affine transportation}

The affine transport is related to covariant derivative of $\mathrm{A}$ which is related to vector cross product $\diamond$ which in turn related to divergence and parallel shift of planes. The orthogonal vectors at $p$ and $Q$ are parallel (null space) we can construct such parallel planes vertically and achieve the same. (Corresponding to $\theta$ and $\eta$ cosystems in $[4,5])$

\section{Theorem}

The weight points corresponding to different differential feedback move along a straight line proof:-The hyper planes, being affine are described in Remanian space by the Levi-civita equation.[3]

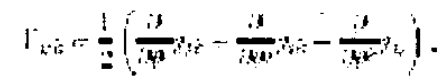

This is the torsion free manifold retaining affine connection. The corresponding geodesic curve is given by[3]

$$
\Gamma \dot{\theta} \dot{\phi}=1
$$

So, 
In the manifold of hyperplanes, the invarianttensor may be defined as

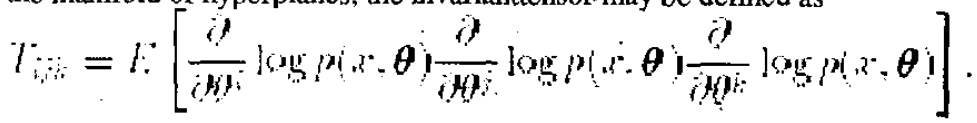

where $p(x,, \theta)$ is the probability function. Using the equation (8) which relates energy and the probability. The equation may be rewritten as

$\mathrm{T}_{\mathrm{ijk}}=\mathrm{E}\left[\Pi \partial(\mathrm{e}) / \partial \theta^{\mathrm{I}}\right]$

e being the energy in the plane as given above The $\propto$ connection is given by

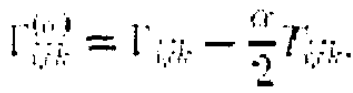

For an affine cosystem, The Riemann-christoffel curvature vanishes, leaving $\alpha=+/-1$. The Geodesic equation reduces to

$\theta(t)=a+b t------(19)$

\section{CONCLUSIONS:}

With differential feedback, it has been found out that the no.. of iterations required for training is reduced. With I order differential feedback, the output represents a plane parallel to original output. The set of differentially fed neural networks form a manifold of parallel planes, with infinite order feedback being the plane with zero error. Also, simulation results show that two terms of II order differential feedback can be replaced by a single equivalent plane. This supports the existence of Eigen plane which substitutes infinite terms of infinite order different feedback.

Since error varies asymptotically with order, the gap between parallel planes decreases and the infinite order plane coincides with Eigen plane. The entropy is minimum when the plane reaches eigen plane. The amount of feedback to be given is equal to kullback divergence. The weight points corresponding to different differential feedback move along a straight line

\section{References}

1.Manjunath.R and K.S.Gurumurthy, System design using differentially fed Artificial Neural networks, IASTED 2002 (accepted)

2. Aarts, E.H.L. and Korst, J.H.M.Simulated Annealing and Boltzmann Machines, Chichester:Wiley.1989

3.S.Amari Information Geometry of the EM and em algorithms for neural networks, Neural networks, 8, No.9,1995

4.Amari.s. Differential geometry of curved exponential families -curvatures and information loss.Annals of statistics.1982

5.Amari.s.Information Geometry of neural networks-New. Bayesian Duality theory-

6.S.Amari,Natural gradient works efficiently in Iearning, Neural computation 10:1998[251-276]

7.S.Amari and H.Nagaoka, Methods of information geometry,AMS and Oxford university press,2000.

8.Information geometry of EM and em algorithms for neural networks, Neural networks $8(9)$ 1995[1379-1408 ] 